ARTICLE

\title{
Cortical idiosyncrasies predict the perception of object size
}

Christina Moutsiana ${ }^{1,2, \star}$, Benjamin de Haas ${ }^{1,3, \star}$, Andriani Papageorgiou1, Jelle A. van Dijk ${ }^{1,3}$, Annika Balraj ${ }^{1,4}$, John A. Greenwood ${ }^{1} \&$ D Samuel Schwarzkopf ${ }^{1,3}$

Perception is subjective. Even basic judgments, like those of visual object size, vary substantially between observers and also across the visual field within the same observer. The way in which the visual system determines the size of objects remains unclear, however. We hypothesize that object size is inferred from neuronal population activity in $\mathrm{V} 1$ and predict that idiosyncrasies in cortical functional architecture should therefore explain individual differences in size judgments. Here we show results from novel behavioural methods and functional magnetic resonance imaging ( $\mathrm{fMRI}$ ) demonstrating that biases in size perception are correlated with the spatial tuning of neuronal populations in healthy volunteers. To explain this relationship, we formulate a population read-out model that directly links the spatial distribution of V1 representations to our perceptual experience of visual size. Taken together, our results suggest that the individual perception of simple stimuli is warped by idiosyncrasies in visual cortical organization.

\footnotetext{
${ }^{1}$ Experimental Psychology, University College London, 26 Bedford Way, London WC1H OAP, UK. ${ }^{2}$ Department of Psychology, Kingston University, Penrhyn Road, Kingston Upon Thames, Surrey, London KT1 2EE, UK. ${ }^{3}$ UCL Institute of Cognitive Neuroscience, 17 Queen Square, London WC1N 3AZ, UK.

${ }^{4}$ Columbian College of Arts and Sciences, The George Washington University, 2125 G Street NW, Washington 20052, USA. * These authors contributed equally to this work. Correspondence and requests for materials should be addressed to D.S.S. (email: s.schwarzkopf@ucl.ac.uk).
} 
$\mathrm{H}$ ow do we perceive the size of an object? A range of recent observations have lent support to the hypothesis that the visual system generates the perceived size of an object from its cortical representation in early visual cortex ${ }^{1}$. In particular, the spatial spread of neural activity in visual cortex has been related to apparent size under a range of contextual modulations ${ }^{2-7}$. The strength of contextual size illusions has further been linked to the cortical territory in V1 that represents the central visual field ${ }^{8,9}$. These findings suggest that lateral connections in V1 may play a central role in size judgments because these interactions are reduced when V1 surface area is larger. Indeed, similar interactions have been argued to underlie the strength of the tilt illusion ${ }^{10,11}$, perceptual alternations in binocular rivalry ${ }^{12}$, the influence of distractors in visual search tasks $^{13}$ and visual working memory capacity ${ }^{14}$. Even the precision of mental imagery co-varies with V1 area ${ }^{15}$ suggesting V1 may be used as a 'workspace' for storing mental images whose resolution is better when surface area is larger.

However, these previous findings do not demonstrate that V1 representations are relevant for size judgments. If $\mathrm{V} 1$ signals were indeed the basis for these judgments, then variations in the functional architecture of V1 should explain idiosyncratic biases in basic size perception (that is, size judgments that occur in the absence of any contextual/illusory effects). To date, this prediction remains untested. Previous neuroimaging experiments have focused on modulations of apparent size that must involve additional processing, either due to local interactions in V1 between adjacent stimuli or by a context that likely involves processing in higher visual areas. Others have shown that the objective ability to discriminate subtle differences between stimuli is related to cortical magnification and spatial tuning in early visual cortex ${ }^{11,16,17}$. However, no experiment to date has shown a relationship between $\mathrm{V} 1$ and subjective perceptual biases in the absence of any contextual interaction, even though it is well established that subjective size judgments for simple, small stimuli can vary substantially between observers and even across the visual field within the same observer.

For instance, previous behavioural research has shown that small visual stimuli appear smaller when they are presented in the periphery ${ }^{18-20}$. A simple explanation for this could be the impoverished encoding of stimuli in peripheral vision. However, when stimuli are dimmed artificially to mimic the peripheral decrease in visibility, the same biases are not found ${ }^{19}$. Another explanation could be that higher brain regions that integrate the perceptual input to V1 into a behavioural decision are poorly calibrated against the decrease in cortical magnification when moving from central to peripheral vision. Small errors in this calibration would cause a residual misestimation of stimulus size based on V1 representations and in turn lead to perceptual misestimation ${ }^{20}$. However, neither of these models can explain why these perceptual biases are consistent underestimates of stimulus size. Impoverished stimulus encoding alone should only result in poorer acuity, while residual errors in calibration would be expected to show both under- and overestimation. Furthermore, recent research has also demonstrated reliable heterogeneity in size judgments across the visual field within individual observers at isoeccentric locations ${ }^{9,21}$ constituting a 'perceptual fingerprint' that is unique to each observer. The neural basis of these individual differences, however, remains unknown.

In the present study, we used functional magnetic resonance imaging (fMRI) to compare perceptual biases in size judgments with individual functional architecture in V1-specifically, the population receptive field ( $\mathrm{pRF}$ ) spread and local cortical surface area. To do so, we developed the Multiple Alternative Perceptual Search (MAPS) task. This approach combines a matching task with analyses similar to reverse correlation ${ }^{22,23}$. Observers search a peripheral array of multiple candidate stimuli for the one whose subjective appearance matches that of a centrally presented reference. This task allows measurement of subjective appearance, while minimizing the decisional confounds present in more traditional tasks like stimulus adjustment or the method of constant stimuli ${ }^{24-26}$. The MAPS task further estimates perceptual biases and discrimination acuity, while several stimuli are presented simultaneously. We consider this a more naturalistic task, akin to our daily perceptual judgments (Fig. 1a), compared with traditional psychophysical tasks involving single, isolated objects.

We show that simple visual stimuli are perceived as smaller when they are encoded by larger pRFs in V1. From this we formulate a population read-out model that directly links the spatial distribution of cortical representations to our perceptual experience of visual size.

\section{Results}

Apparent size depends on eccentricity. Thirteen normal, healthy observers viewed an array of five circles on each trial and made a perceptual judgment (Fig. 1b). The central circle was constant in size and served as the reference. Observers reported which of the four target circles appeared most similar in size to the reference. We fit a model to explain each observer's behavioural responses, with each of the four target locations modelled via the output of a detector tuned to stimulus size. In each trial the detector showing the strongest response was used to predict the observer's behavioural choice. This procedure allowed the estimation of both raw perceptual bias and uncertainty (dispersion) at each location (Fig. 1c).

Peripheral stimuli appeared smaller on average than the central reference, confirming earlier reports ${ }^{18,20,27}$. This reduction in apparent size increased with stimulus eccentricity (Fig. 1d, black curve). When instead of isolated circles we presented the target circles inside larger concentric circles, perceptual biases were predictably shifted in the other direction (the Delboeuf illusion ${ }^{28}$ ) so that targets appeared on average larger than the reference (Fig. 1d, red curve). This illusory effect interacted with the effect of eccentricity on apparent size, leading again to a gradual reduction in (illusory) size as stimuli moved into the periphery. This differs somewhat from the classical Delboeuf illusion, where perceptual biases are typically compared with a reference either at the same eccentricity or even at the same stimulus location. In contrast, in our task the reference is at fixation. To disentangle the illusion from the effect of eccentricity, we therefore also calculated the relative illusion strength, that is, the difference in perceptual bias for isolated circles and the illusion stimuli at each location. This effect (here an increase in apparent size) also increased with eccentricity (Fig. 1d blue curve; but note that since observers never compared the stimuli directly at isoeccentric locations this may not fully account for the classical Delboeuf illusion). To summarize, objects appear increasingly smaller as they move into peripheral vision, where the magnitude of size illusions also has an increasing effect (here with the Delboeuf illusion to make them appear larger).

These results cannot be trivially explained by differences in discrimination acuity. Because spatial resolution decreases in peripheral vision, it is theoretically possible that bias estimates are noisier at greater eccentricities and thus produce this pattern of results, in particular for the Delboeuf stimuli where bias magnitude decreases. To rule out this confound we also calculated mean bias estimates weighted by the precision of observers' size estimates (that is, the reciprocal of dispersion) at the corresponding locations. The pattern of results is very similar to the one for 
a

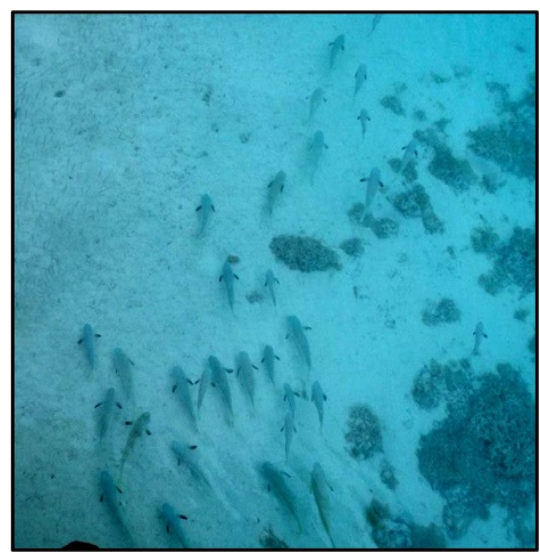

b

C
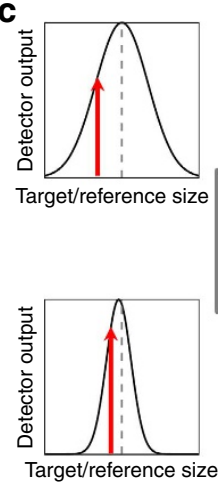

Target/reference size

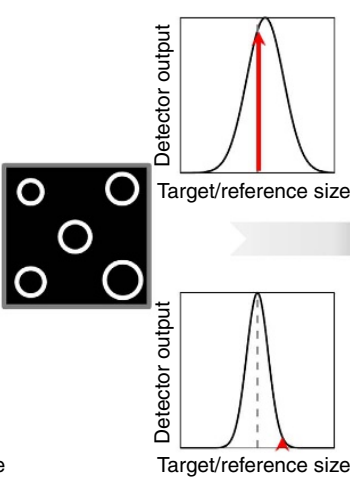

d

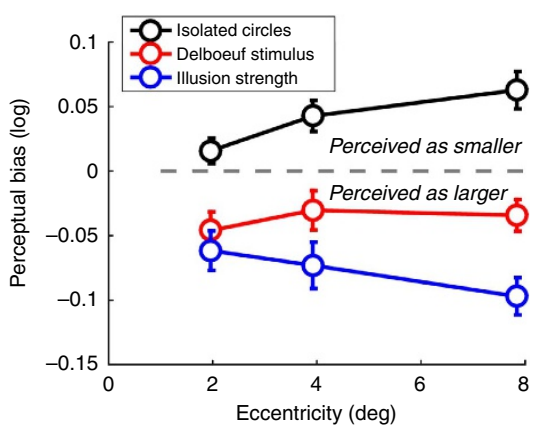

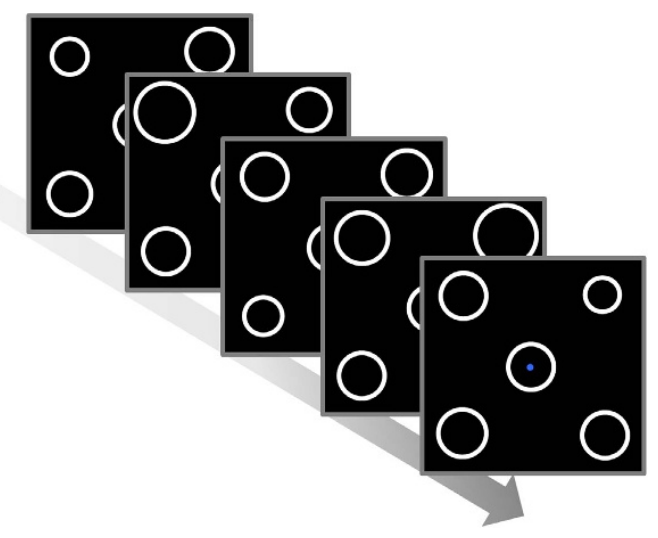

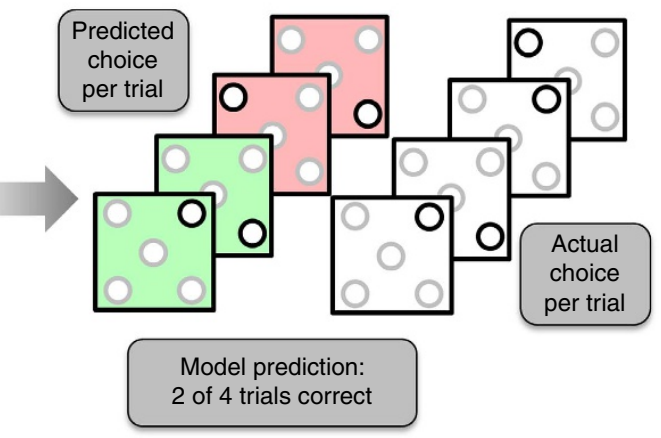

e

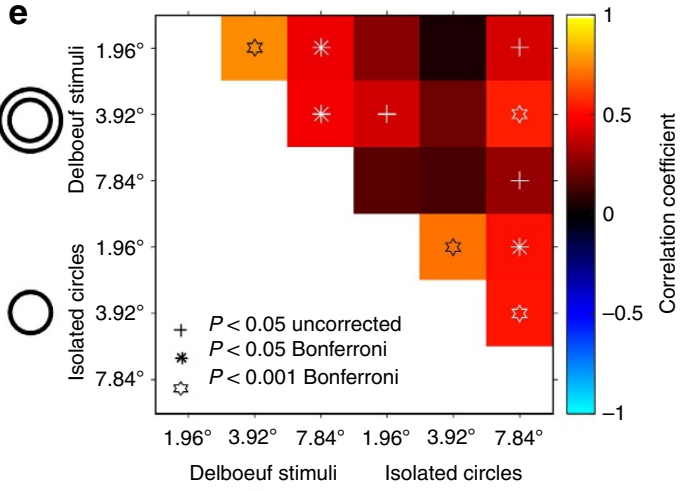

Figure 1 | Idiosyncratic biases in size perception. (a). Visual objects often appear in the presence of similar objects. For example, a spearfisherman may be searching this school for the largest fish. What is the neural basis for this judgment? (b). The MAPS task. In each trial, observers fixated on the centre of the screen and viewed an array of five circles for $200 \mathrm{~ms}$. The central circle was constant in size, while the others varied across trials. Each frame here represents the stimulus from one trial. The arrow denotes the flow of time. Observers judged which of the circles in the four corners appeared most similar in size to the central one. (c). Analysis of the behavioural data from MAPS task. The behavioural responses in each trial were modelled by an array of four 'neural detectors' tuned to stimulus size (expressed as the binary logarithm of the ratio between the target and the reference circle diameters). Tuning was modelled as a Gaussian curve. The detector showing the strongest output to the stimulus (indicated by the red arrows) determined the predicted behavioural response in each trial (here, the top-right detector would win). Model-fitting minimized the prediction error (in this example the model predicted the actual behavioural choice correctly for $50 \%$ of trials) across the experimental run by adapting the mean and dispersion of each detector. (d). Average perceptual bias (positive and negative: target appears smaller or larger than reference, respectively), across individuals plotted against target eccentricity for simple isolated circles (black), contextual Delboeuf stimuli (red), and relative illusion strength (blue), that is, the difference in biases measured for the two stimulus conditions. Error bars denote \pm 1 s.e.m. (e). Correlation matrix showing the relationship of unique patterns of perceptual biases in the two conditions (isolated circles and Delboeuf stimuli) and at the three target eccentricities. Colour code denotes the correlation coefficient. Symbols denote statistical significance. Crosses: $P<0.05$ uncorrected. Asterisks: $P<0.05$ Bonferroni corrected. Hexagrams: $P<0.001$ Bonferroni corrected.

raw biases (Supplementary Fig. 1a). Two years after the initial experiment, we also conducted another small experiment on four observers. In this experiment we included two larger eccentricities $\left(11.76^{\circ}\right.$ and $\left.15.68^{\circ}\right)$. This confirmed that the size of isolated circles continue to be underestimated even at larger eccentricities. In contrast, although the size of Delboeuf stimuli is overestimated 
a

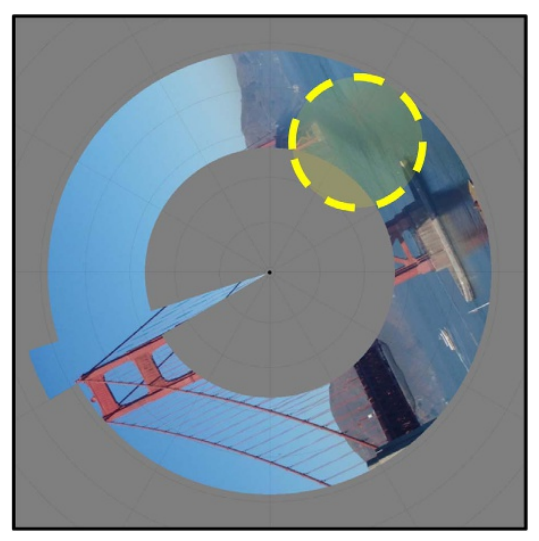

b
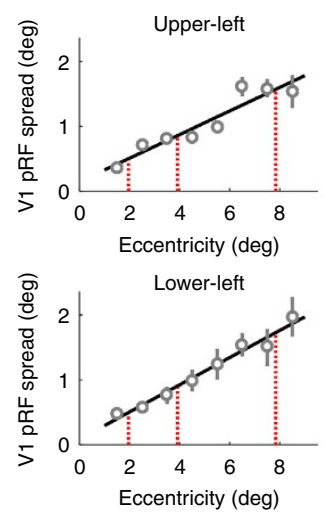
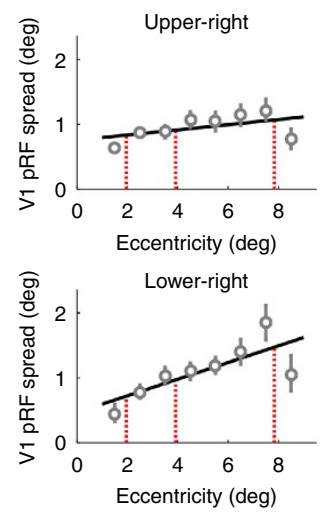

C

d
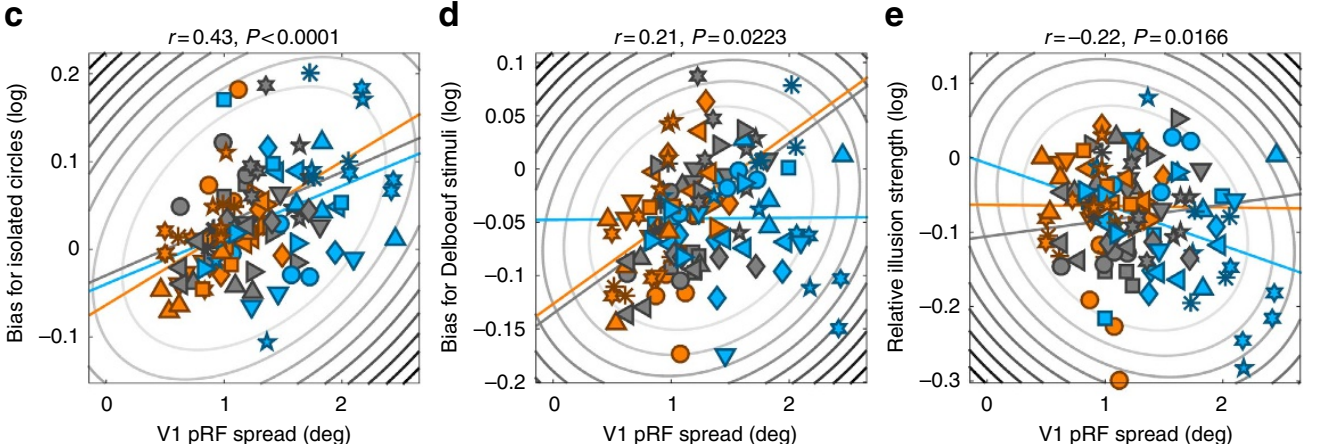

O

f
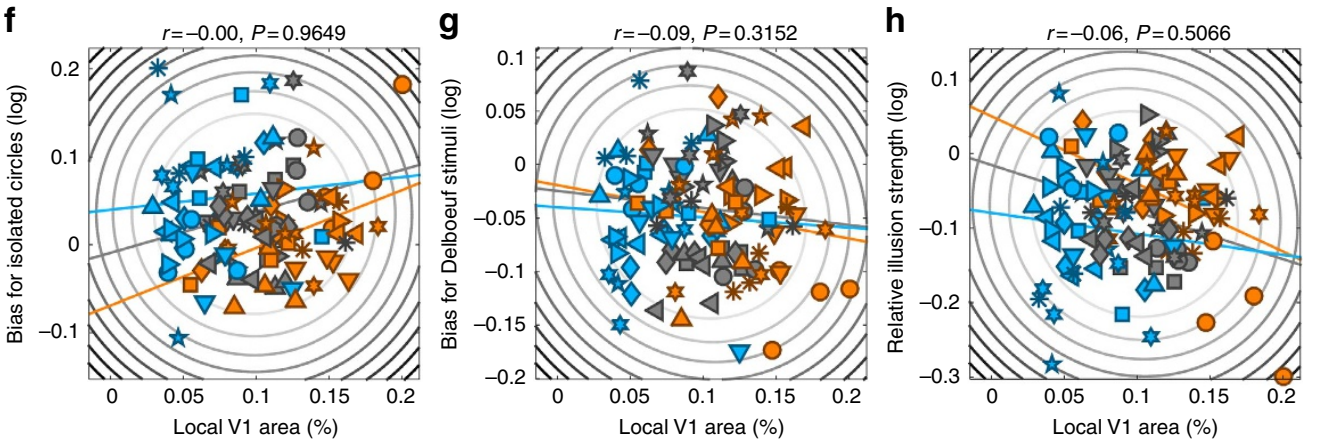

Figure 2 | Neural correlates of size perception. (a). Population receptive field (pRF) mapping with fMRI. Observers viewed natural images presented every $500 \mathrm{~ms}$ within a combined wedge-and-ring aperture. In alternating runs the wedge rotated clockwise/counterclockwise in discrete steps $(1 \mathrm{~Hz})$ around the fixation dot while the ring either expanded or contracted. A forward model estimated the position and size of the pRF (indicated by yellow circle) that best explained the fMRI response to the mapping stimulus. (b). We estimated the pRF spread corresponding to each target location in the behavioural experiment by fitting a first-order polynomial function (solid black line) to pRF spreads averaged within each eccentricity band for each visual quadrant and extrapolating the pRF spread at the target eccentricities. Grey symbols in the four plots show the pRF spread by eccentricity plots for the four target locations (see insets) in one observer. Grey error bars denote bootstrapped 95\% confidence intervals. The solid black line shows the polynomial fit used to estimate pRF spread at each target location. The vertical red dashed lines denote the three stimulus eccentricities at which we extrapolated pRF spread and surface area from the fitted polynomial functions. Data from other observers and V1 surface area measurements are included as Supplementary Information. (c-e). Perceptual biases for isolated circles (c), Delboeuf stimuli (d), and the relative illusion strength (e) plotted against pRF spread for each stimulus location and observer. (f-h). Perceptual biases for isolated circles (f), Delboeuf stimuli ( $\mathbf{g}$ ), and the relative illusion strength (h) plotted against V1 surface area (as percentage of the area of the whole cortical hemisphere) for each stimulus location and observer. In $\mathbf{c}-\mathbf{i}$, symbols denote individual observers. Elliptic contours denote the Mahalanobis distance from the bivariate mean. The coloured, straight lines denote the linear regression separately for each eccentricity. Colours denote stimuli at $1.96^{\circ}$ (orange), $3.92^{\circ}$ (grey), or $7.84^{\circ}$ (light blue) eccentricity.

at the more central eccentricities, the bias magnitude decreases with eccentricity and is close to zero at the most peripheral location tested (Supplementary Fig. 1b). However, the accuracy of performing the MAPS task also decreases with eccentricity, especially for Delboeuf stimuli (Supplementary Fig. 1c)), likely because both resolution and crowding ${ }^{29}$ increasingly disrupt these judgments of size.
Idiosyncratic biases in size perception. Critically, we next analysed the idiosyncratic pattern of perceptual biases for each observer by comparing biases across the visual field, for both isolated circles and Delboeuf stimuli. To do so, bias estimates were taken from all observers in each visual field quadrant, separately for each eccentricity and stimulus type (that is, each visual field location was treated as a separate data point, so 

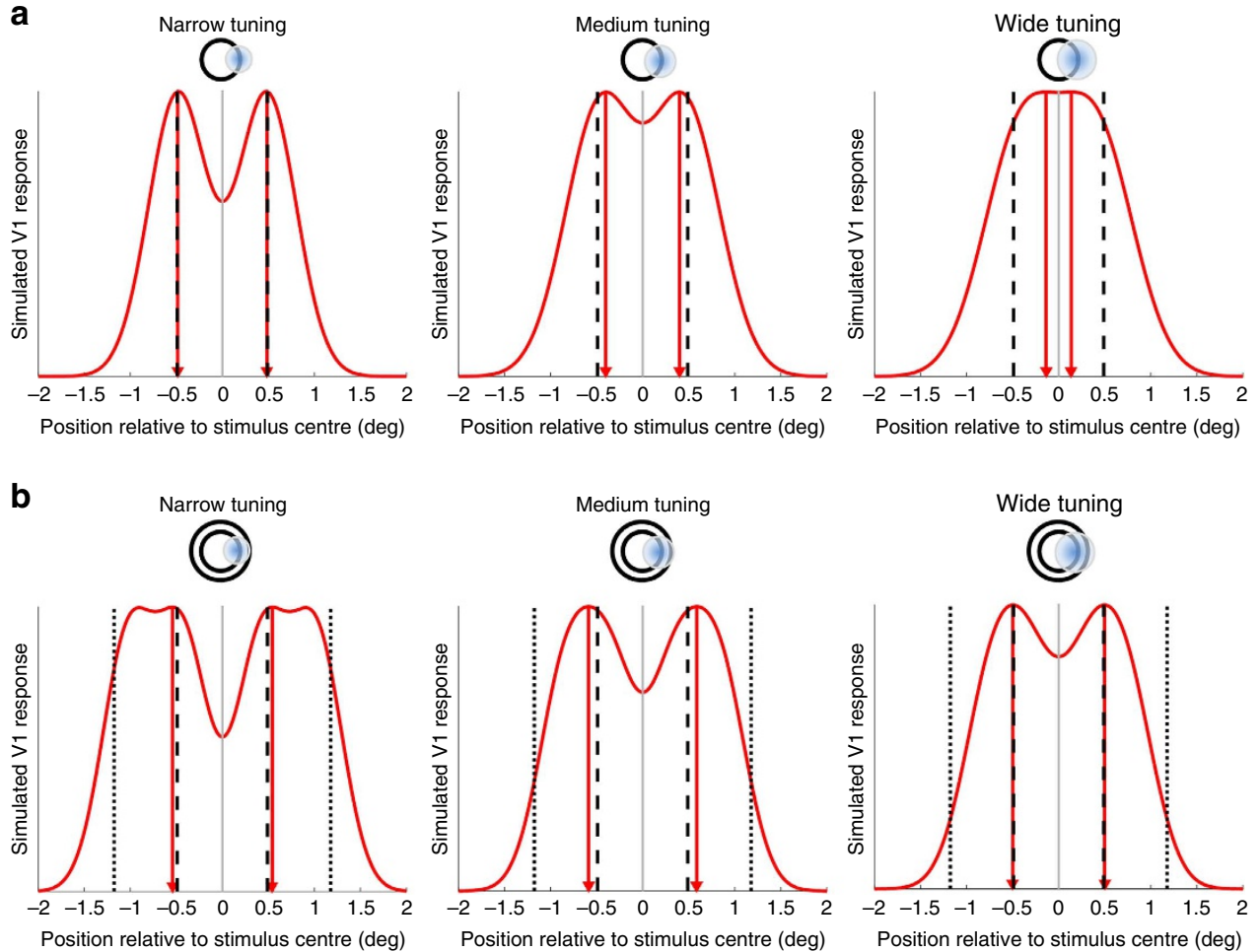

Figure 3 | Basic read-out model. (a,b). Simulated activity profiles for isolated circle (a) and Delboeuf stimuli (b) were generated by passing the actual location of the stimulus edges through a bank of Gaussian filtres covering the stimulus locations. The red curves indicate the output of the filter bank as a simulation of stimulus-related population activity in V1. The separation between the two peaks is an estimate of stimulus size (red triangles and vertical red lines). The vertical black lines denote the actual position of the edges of the target stimulus (dashed) and the inducer annulus in the Delboeuf stimuli (dotted). Simulated neuronal tuning width increases from left to right (see also the schematic diagram above each graph representing the stimulus and an example receptive field).

$n=40)$. Biases were strongly correlated across both stimulus type and over the three eccentricities tested (Fig. 1e; Supplementary Fig. 2). That is, if observers perceived a strong reduction in the apparent size of a stimulus at a given location, then they tended to show strong reductions for the same stimulus type within the same visual field quadrant, regardless of eccentricity. Variations between different quadrants of this kind are consistent with the anatomical separation of visual quadrant maps in retinotopic areas. Psychophysical studies suggest that similarly coarse differences may be common, for instance, with the frequent observation that performance in the lower visual field exceeds that in the upper visual field ${ }^{30,31}$. We further confirmed that these bias estimates were highly reliable even between testing sessions separated by 1 or 2 years (Supplementary Note 1 ).

Perceptual biases correlate with spatial tuning. Next we employed fMRI with pRF mapping to estimate the tuning of V1 voxels to spatial position (Fig. 2a,b (refs 32-34)). Importantly, these neuroimaging experiments were independent from the behavioural experiments and conducted many months later in a different testing environment (MRI scanner versus behavioural testing room).

Interestingly, this analysis revealed a systematic relationship between perceptual biases and pRF spread (also known as pRF size or the $\sigma$ parameter of the pRF model). With the data averaged across observers, increasing eccentricity gives both an increase in pRF spread ${ }^{32}$ (Supplementary Data 1) and a decrease in apparent size (Fig. 1d). We then considered the individual data by calculating a linear multiple regression model using pRF spread to predict perceptual bias at each of the 120 locations
(12 locations in 10 observers). Separate regressors accounted for the between-subject variance (mean per observer), the individual eccentricity effects (mean per eccentricity for each observer) and the individual effects of the visual field quadrant (mean per quadrant for each observer). Both individual eccentricity $(\beta=0.05, t(116)=3.09, P=0.0025)$ and quadrant $(\beta=0.06$, $t(116)=2.61, P=0.01)$ effects were significant predictors of the biases for isolated circles. The between-subject variance effect was not significant $(\beta=-0.05, t(116)=-1.04, P=0.2175)$. That is, across different locations, stronger decreases in apparent size were associated with larger pRFs. For Delboeuf stimuli, none of these effects were significant (eccentricity: $\beta=0.0, t(116)=-0.06$, $P=0.9493 ; \quad$ quadrant: $\quad \beta=0.02, \quad t(116)=0.69, \quad P=0.4946$; between-subject variance: $\beta=0.05, t(116)=1.52, P=0.1322$ ).

Next, we considered every stimulus location in every observer as a separate observation and calculated the correlation between pRF spread and perceptual biases. Both isolated circles (Pearson's $r=0.43, \quad P<0.0001, n=120$; Fig. 2c) and Delboeuf stimuli ( $r=0.21, P=0.0223, n=120$; Fig. $2 \mathrm{~d}$ ) were perceived as smaller when they were presented at visual field locations covered by voxels with larger pRFs. We obtained similar results when analysing the data separately for each eccentricity, except for the Delboeuf stimuli at the largest eccentricity (Supplementary Fig. 3a,b). These individual differences demonstrate that idiosyncratic variations in pRFs correlate with apparent size at a fixed eccentricity. Our relative illusion strength also showed a negative correlation with $\mathrm{pRF}$ spread $(r=-0.22, P=0.0166, n=120$; Fig. 2e), indicating that larger pRFs were associated with smaller differences between raw biases for Delboeuf stimuli and isolated circles. This result was, however, largely driven by the results for the largest eccentricity (Supplementary Fig. 3c). 
These correlation analyses include the between-subject variance as well as the pattern of differences within each observer and therefore directly quantify the relationship between the two variables. However, the measurements from the four visual field quadrants for a given observer are naturally not independent. We therefore conducted several additional tests. We first repeated all of these analyses after subtracting the mean bias/pRF spread from each observer and eccentricity. This allows analysis of the pattern of results across quadrants, while removing both the individual differences between observers (between-subject variance) and differences related to eccentricity. This analysis confirmed the correlation between pRF spread and biases for isolated circles (Pearson's $r=0.29, P=0.001, n=120$ ). For Delboeuf stimuli and the relative illusion strength the correlations were not significant, though they showed the same trends as the equivalent correlations in the main analysis (Delboeuf stimuli: $r=0.15$, $P=0.112, \quad n=120$; illusion strength: $r=-0.16, \quad P=0.077$, $n=120)$. We also conducted a similar second-level analysis, in which we first calculated the correlations across the four locations separately in each observer at each eccentricity and then tested whether the average correlation (after $z$-transformation) was significantly different from zero (see Supplementary Note 2 for more detail on these analyses and Supplementary Tables 1 and 2 for full results).

A similar approach exploiting within-subject correlations has previously been used in the context of the retinotopic mapping data $^{35,36}$ and spatial heterogeneity in perceptual function ${ }^{21}$. These studies suggest that our sample size of 10 observers is likely sufficient. However, to confirm this we also performed a simulation analysis to quantify the statistical power of our approach. Both our main analysis and the additional analysis with between-subject variance removed had the greatest sensitivity for detecting a true effect (with approximate power of $90 \%$ for an assumed true correlation of $r=0.3$ ). This is unsurprising given the large number of data points in these analyses $(n=120)$. However, while all other analyses produced nominal false positive rates of $\sim 5 \%$, false positives rose slightly to $\sim 9 \%$ when removing between-subject variance. This suggests our main analysis as the optimal statistical test for our hypothesis, affording high sensitivity and specificity (Supplementary Note 3).

Finally, we also analysed the equivalent correlations between behavioural measures and pRF spread for areas V2 and V3. Pooled across eccentricities pRF spreads in both areas were significantly correlated with the biases for isolated circles (V2: Pearson's $r=0.4, P<0.0001, n=120$; V3: $r=0.29, P=0.0013$, $n=120$ ). Correlations between pRF spread in these areas and the

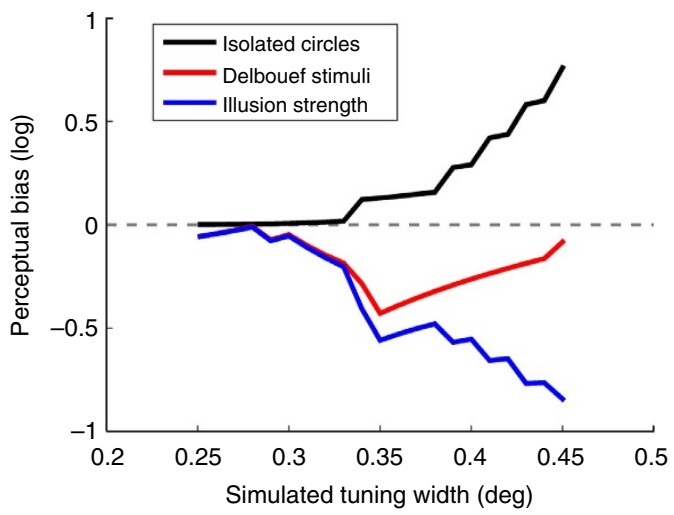

Figure 4 | Biases from read-out model. Simulated perceptual biases for the two stimulus types and the relative illusion strength plotted against assumed tuning width. Black: isolated circles. Red: Delboeuf stimuli. Blue: relative illusion strength. See Fig. 1d for comparison. biases for Delboeuf stimuli followed the same trend but were not significant (V2: $r=0.14, P=0.1188, n=120$; V3: $r=0.16$, $P=0.0728, n=120)$. Moreover, separated by eccentricity all of these correlations were positive but not significant. Thus, the relationship between pRF spread and perceptual biases was not specific to V1 but a general feature of early visual cortex. This is unsurprising given that pRF spreads in V1 were largely well correlated with the extrastriate regions (minimal correlation, separately for each eccentricity in V2: Pearson's $r=0.49$, $P=0.0014, n=40$; V3: $r=0.26, P=0.1037, n=40)$.

Basic read-out model of size perception. Why should the apparent size of our circle stimuli be smaller when pRFs are larger? While this result is consistent with the simple impoverishment hypothesis, which states that perceptual biases depend on the precision of the stimulus representation, this alone does not explain why biases are consistent underestimates of stimulus $\operatorname{size}^{19}$. We therefore conducted a series of simulations that assume that higher brain regions involved in integrating sensory inputs into a perceptual decision about object size read out signals from $\mathrm{V} 1$ neurons $^{37}$. We simulated the neuronal activity inside the retinotopic map by passing the actual spatial position of the two edges of the stimulus through a Gaussian filter bank covering that stimulus location. The stimuli were simulated as a binary vector representing 1,050 pixels (corresponding to the height of the screen) where the edges were set to 1 , while the background was set to 0 . The filter bank assumed a Gaussian tuning curve whose width was parameterized at each pixel along this vector. We calculated the response of each filtre, to give rise to a population activity profile. Subsequently, a higher level then sampled this activity to infer stimulus size. For simplicity, this basic model only assumes two layers but it is principally the same, if activity is submitted from V1 across multiple stages along the visual hierarchy.

With this approach, stimulus size can be inferred from the distance between the activity peaks corresponding to the two edges (Fig. 3). When the spatial tuning of visual neurons (that is, neuronal receptive field size) is narrow, the peaks can accurately localize the actual stimulus edges. However, as tuning width increases, the activity profile becomes blurrier. Critically, the distance between the two peaks also becomes smaller because activity from the two edges is conflated (Fig. 3a). It follows that with wider tuning (at greater eccentricity and larger pRF spread), estimates of the separation between peaks decreases until eventually the two peaks merge. This scenario presumably corresponds to far peripheral vision. For the Delboeuf stimuli, the separation of peaks is greater than that of the actual stimulus edges when tuning width is narrow because activity corresponding to the inducer and the target blurs together. However, as tuning width increases the separation also becomes smaller just as for isolated circles (Fig. 3b).

To quantify the perceptual biases predicted by this model, we simulated perceptual judgments for both stimulus types and across a range of neuronal tuning widths. The relationship between increasing tuning width and perceptual biases parallels that between empirically observed perceptual biases and stimulus eccentricity (Fig. 4). Size estimates at very small receptive fields (and thus lower eccentricity) are largely accurate, but apparent size becomes increasingly smaller than the physical stimulus as tuning width increases. Estimates for the Delboeuf stimuli are generally larger than the physical target. However, as tuning width increases estimates again become smaller. Thus, a large part of the difference in perceptual quality between these two stimulus types may be simply due to the physical difference between them, and the corresponding representation within a population of 

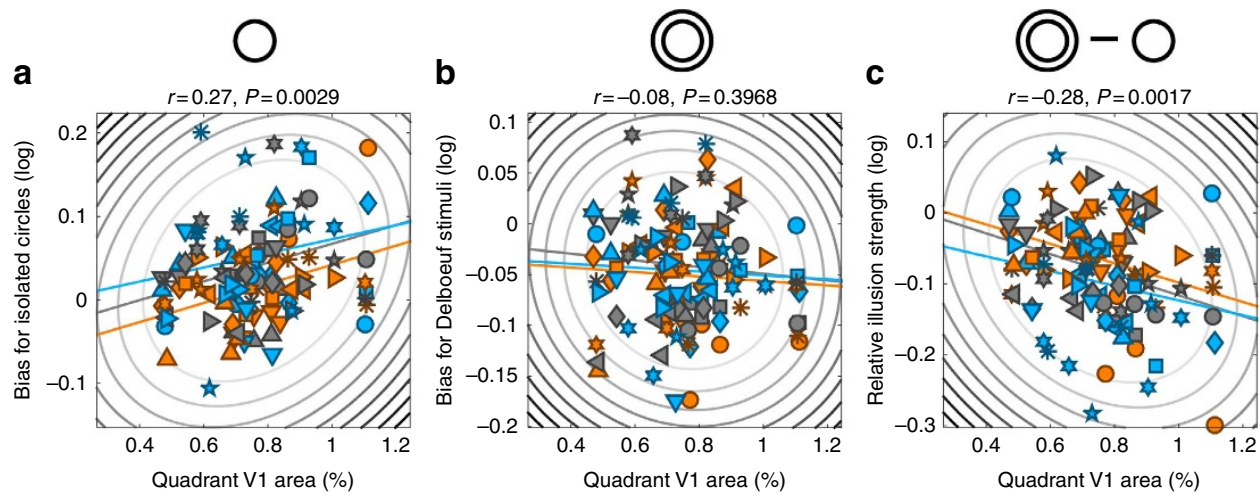

Figure 5 | Correlations with V1 surface area. Perceptual biases for isolated circles (a), Delboeuf stimuli (b) and the relative illusion strength (c) plotted against the surface area (as percentage of the whole cortex) for each quadrant map in $\mathrm{V} 1$ between $1^{\circ}$ and $9^{\circ}$ eccentricity and observer. Symbols denote individual observers. Elliptic contours denote the Mahalanobis distance from the bivariate mean. The coloured, straight lines denote the linear regression separately for each eccentricity. Colours denote stimuli at $1.96^{\circ}$ (orange), $3.92^{\circ}$ (grey) or $7.84^{\circ}$ (light blue) eccentricity.

receptive fields, rather than a more complex interaction between the target and the surrounding annulus. Finally, our relative illusion strength is the difference between biases for the two stimulus types. As tuning width increases, this measure in turn becomes larger, just as it does for the empirical data in Fig. $2 \mathrm{~d}$.

One caveat to this model is that the magnitude of simulated biases is a lot larger than those we observed empirically. This may indicate additional processes involved in calibrating the size judgment. However, it may also be simplistic to infer size from the actual activity peaks. The actual read-out process may instead calculate a confidence range that accounts for the whole function describing the activity profile ${ }^{38}$. The exact relationship between pRF spread and neuronal receptive field size is also unknown. Estimates of $\mathrm{pRF}$ spread from the fMRI data must aggregate the actual sizes of neuronal receptive fields, but also the range of centre positions of all the receptive fields in the voxel, and their local positional scatter within this range. In addition, extra-classical receptive field interactions, response nonlinearities ${ }^{39}$, and nonneuronal factors like hemodynamic effects, fixation stability and head motion must also contribute to some extent. While the simulated tuning widths in our model probably roughly correspond to neuronal receptive field size in V1 within our eccentricity range $\mathrm{e}^{32}$, an aggregate of the different factors contributing to pRF spread may thus be more appropriate. However, at least qualitatively the relationship between perceptual biases and tuning width parallels the empirical pattern of perceptual biases and pRF spread estimates that we found.

Delboeuf illusion strength correlates with V1 surface area. At the smallest eccentricity of $1.96^{\circ}$, raw perceptual biases for isolated circles were correlated with local V1 area but this pattern was not evident when the data were pooled across eccentricity (Pearson's $r=-0.0, \quad P=0.9649, \quad n=120$; Fig. $2 \mathrm{f}$ and Supplementary Fig. 4a). With Delboeuf stimuli, raw perceptual biases (relative to the central reference) did not correlate with local V1 area at any eccentricity $(r=-0.09, P=0.3152, n=120$; Fig. $2 \mathrm{~g}$ and Supplementary Fig. 4b). Because previous research compared perception with the macroscopic surface area of the entire central portion of V1 (refs 8,9,11-15,17), we further calculated the overall surface area of $\mathrm{V} 1$, representing each visual field quadrant between an eccentricity of $1^{\circ}$ and $9^{\circ}$. This showed a similar relationship with perceptual biases as local V1 area (Fig. 5) for isolated circles (Pearson's $r=0.27, P=0.0029$ ) but not Delboeuf stimuli $(r=-0.08, P=0.3968)$. The results further suggest that the variability in perceptual biases is largely driven by differences in cortical magnification for the central visual field since for our innermost eccentricity the relationship between surface area and perceptual measures was always strongest. This variability in central V1 area may thus dominate measurements of the whole quadrant. However, the macroscopic surface area should also be a more stable measure than the area of small local cortical patches. The local surface area and overall area of quadrant maps were very strongly correlated (area relative to whole cortex: Pearson's $r=0.54, P<0.0001$; absolute surface area: $r=0.54, P<0.0001$; see Fig. 6 for plots separated by eccentricity). Therefore, the macroscopic V1 surface area is a close proxy for local variations in cortical magnification.

In an indirect replication of our earlier findings ${ }^{8,9,11}$, we also observed an inverse relationship between the relative strength of the Delboeuf illusion (the difference in perceptual bias measured for the two stimulus types) and V1 surface area. Again, this was only significant at the smallest eccentricity and not when the data were pooled across eccentricities (Pearson's $r=-0.06$, $P=0.5066$; Figs $2 \mathrm{~h}$ and $5 \mathrm{c}$ ) but it was significant for the overall area of the quadrant map $(r=-0.28, P=0.0017)$. The relative illusion strength (and thus presumably the Delboeuf illusion itself) is the difference in apparent size between these stimuli at the same location. This measure could be partially independent of pRF spread, as it may instead be related to long-range horizontal connections that exceed the voxel size and that mediate the contextual interaction between target and surround.

Under the hypothesis that surface area predicts illusion strength, the bias induced by the illusion differs mechanistically from basic perceptual biases. Both isolated circles (Fig. 2c) and Delboeuf stimuli (Fig. 2d) were perceived as smaller when pRFs were larger. However, at the same location Delboeuf stimuli were nonetheless seen as larger than isolated circles. Even though the apparent size of both isolated circles and Delboeuf stimuli was linked to pRF spread-consistent with the basic read-out modelthe difference between these biases was also modestly correlated with the area of central V1. The illusion effect may be modulated by cortical distance, possibly via lateral intracortical connections ${ }^{1,10}$, rather than $\mathrm{pRF}$ spread. We conjectured previously that the illusion could arise due to long-range connectivity between V1 neurons encoding the target circle and the surrounding context. Thus, the illusion may be weaker when V1 surface area (and thus cortical distance) is larger ${ }^{8-11}$. In contrast, basic perceptual biases for any stimulus seem to be linked to the coarseness ( $\mathrm{pRF}$ spread) of the retinotopic stimulus representation itself, which relates to neuronal receptive field sizes and their local positional scatter.

This interpretation may seem to contradict previous findings that pRF spread is inversely related to V1 surface area ${ }^{17,36}$. 
a

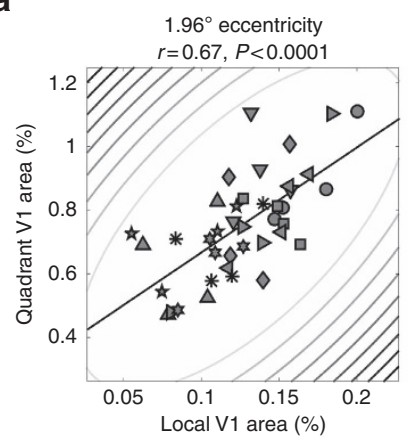

b

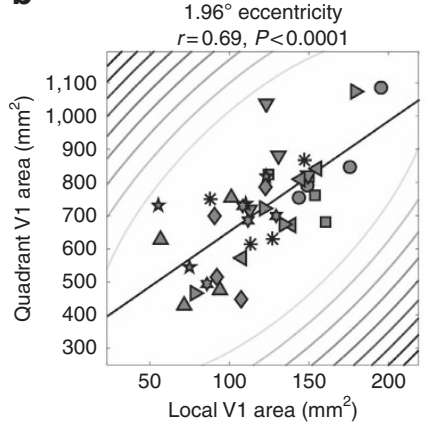

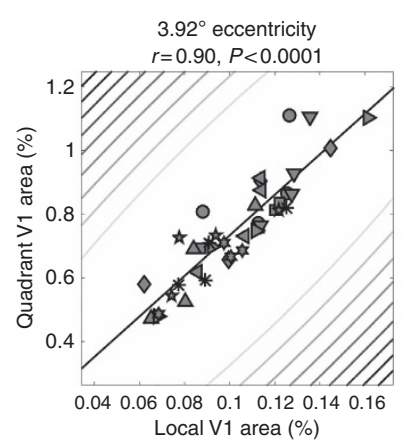
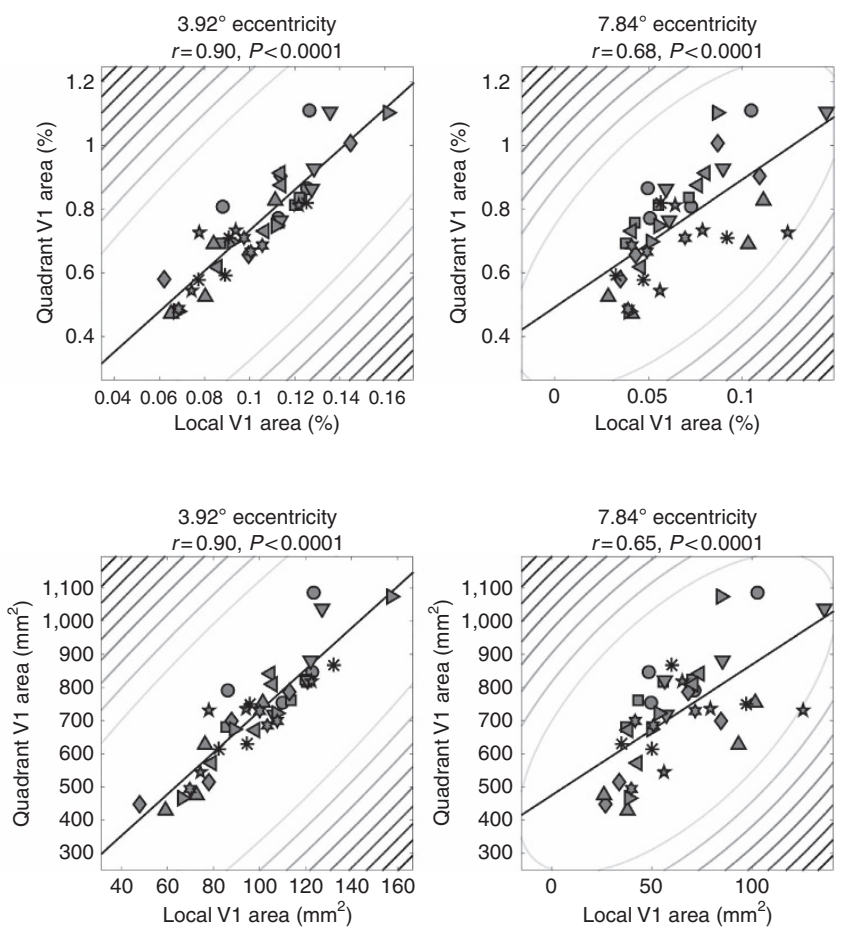

Figure 6 | Comparing local and quadrant area. The surface area of the whole quadrant map in $\mathrm{V} 1$ between $1^{\circ}$ and $9^{\circ}$ eccentricity and each observer plotted against the surface area of the corresponding location in V1 for each observer and target stimulus location. Surface area is expressed either as a percentage of the whole cortical hemisphere (a) or as absolute area (b). Columns show the data for stimuli at $1.96^{\circ}, 3.92^{\circ}$, or $7.84^{\circ}$ eccentricity. Symbols denote individual observers. Elliptic contours denote the Mahalanobis distance from the bivariate mean. The straight, black lines denote the linear regression.

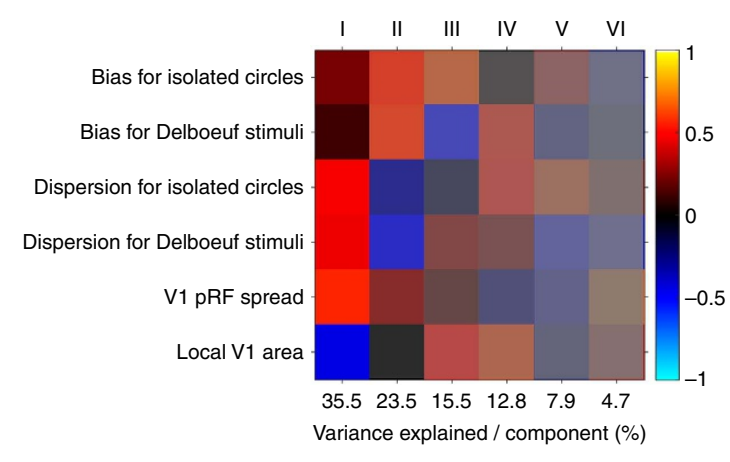

Figure 7 | Principal component analysis. We combined a data set comprising perceptual biases for isolated circles and Delboeuf stimuli, their respective dispersions (that is, discrimination acuity), V1 pRF spread, and local V1 surface area (relative to the area of the whole cortical hemisphere). Columns indicate the six principal components. The numbers on the $x$ axis show the percentage of variance explained by each component. Each row is one of the six variables. The colour of each cell denotes the sign (red: positive; blue: negative) and magnitude of how much each variable contributes to each principal component. The saturation denotes the amount of variance explained by each principal component.

However, there is considerable additional unexplained variance to this relationship. To further disentangle the potential underlying factors, we conducted a principal component analysis on a multivariate data set, including $z$-standardized raw biases for isolated circles and Delboeuf stimuli, the respective dispersions of these distributions (as an indicator of discrimination thresholds), and $\mathrm{pRF}$ spread estimates as well as local surface area at corresponding locations in V1. The first four components explained over $87 \%$ of the variance (Fig. 7). The first component suggests a positive relationship between pRF spread and dispersion and a negative relationship with V1 area. This supports earlier findings linking pRF spread and cortical magnification to acuity ${ }^{16,17}$. There is, however, little relation between these measures and perceptual biases. The second component shows a positive relationship between biases for both stimulus types and pRF spread, which reflects our present results (Fig. 2c,d). In contrast, the third component involves a negative correlation between biases for the two stimulus types and a positive link between raw biases for isolated circles and V1 area. This may explain the negative correlation between relative illusion strength and V1 area (Supplementary Fig. 4c). The fourth component involves a positive link between dispersion for isolated circles and biases for Delboeuf stimuli and also with V1 area. This resembles our earlier findings for orientation processing that also suggest a link between discrimination thresholds for isolated grating stimuli, the strength of the contextual tilt illusion and V1 surface area ${ }^{11}$.

Our results indicate that different mechanisms influence apparent size: both isolated circles and Delboeuf stimuli generally appear smaller (relative to the central reference) when pRFs are large, as predicted by the read-out model. However, variability in cortical surface area (and thus the scale required of intracortical connections) also seems to be an important factor in the illusory modulation of apparent size. Because our task estimates perceptual biases under either condition relative to a constant reference, it was uniquely suited to reveal dissociations between these effects. A more traditional task in which reference stimuli are presented at matched locations/eccentricities would be insensitive to this difference.

Heterogeneity in perceptual biases has central origin. Naturally, the spatial heterogeneity in perceptual biases could possibly arise from factors before visual cortical processing, like small corneal 


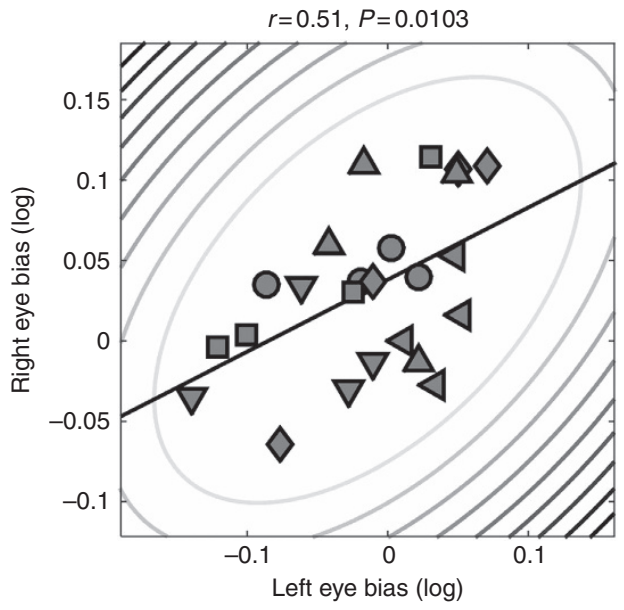

Figure 8 | Perceptual biases measured under dichoptic presentation.

Biases measured with stimuli in the left eye plotted against those measured in the right eye. Symbols denote individual observers. Elliptic contours denote the Mahalanobis distance from the bivariate mean. The straight, black lines denote the linear regression.

aberrations, inhomogeneity in retinal organization or the morphology of retinotopic maps in the lateral geniculate nucleus. We tested this possibility in a behavioural control experiment in which we measured perceptual biases, while we presented the stimuli either binocularly or dichoptically to the left and right eye. There was a close correspondence between biases measured with either eye (Pearson's $r=0.51, P=0.0103$; Fig. 8). Thus, at least a large part of the variance in perceptual biases must arise at a higher stage of visual processing where the input from both eyes has converged, such as the binocular cells in V1.

\section{Discussion}

Our experiments show that when the spatial tuning of neuronal populations in V1 is coarse, visual objects are experienced as smaller. These findings support the hypothesis that object size is inferred by decision-making processes from the retinotopic representations in early visual cortex ${ }^{1}$ and consistent with previous reports of a neural signature of apparent size in V1 responses $^{2-7}$. Our experiments provide strong evidence that the representation in early visual cortex is indeed used for perceptual decisions about stimulus size, because the biases we measured were independent from contextual or top-down modulation of early visual cortex. Considering that perceptual biases correlate with cortical measures acquired a year later and under completely independent conditions (MRI scanner versus behavioural testing room) we posit that this link between cortical measures and perception is a stable feature of the human visual system.

We have formulated a basic read-out model that samples the activity in early retinotopic maps to infer stimulus size. This model predicts the relationship we observed between eccentricity, pRF spread and raw perceptual biases measured behaviourally. This was true both for simple, isolated circle stimuli and the Delboeuf stimuli in which the target was surrounded by an annulus. Taking advantage of our unique task design, we further demonstrate that processes related to basic perceptual biases are dissociable from contextual effects, like the Delboeuf illusion: while raw perceptual biases of object size are explained by $\mathrm{pRF}$ spread, the local surface area (a measure of cortical distance) of at least the part of V1 representing the very central visual field also explains some variance in contextual modulation of apparent size in these illusions. This underlines the need for a greater understanding of how cortical distance relates to pRF spread. Note, however, that we only calculated the relative strength of the Delboeuf illusion based on the biases measured for the two stimulus types, isolated circles and the contextual stimulus including an annulus. It is possible that this prediction does not fully account for the illusion strength one would measure in more standard procedures.

An alternative explanation to our read-out model is that higher-level decoding mechanisms misestimate the size of the stimulus, because they are inadequately calibrated to idiosyncratic differences in cortical magnification ${ }^{20}$. This would cause a residual error between the grossly calibrated read-out that may be reflected in perceptual judgments. This explanation, however, does not explain why perceptual biases are consistently reductions in apparent size. In contrast, our basic read-out model fully accounts for this pattern of results. However, we do not wish to rule out the calibration error hypothesis entirely and in fact a hybrid of the two is certainly possible. In particular, in foveal vision, where individual differences in cortical magnification are far more pronounced ${ }^{33}$, calibration errors are likely. Moreover, the perceptual biases predicted by our basic model are considerably larger than those we observed empirically (even though the relationship with eccentricity parallels the observed data). This finding is consistent with a calibration mechanism that compensates for the incorrect estimation based on basic read-out of the activity in V1.

Naturally, absence of evidence is not evidence of absence. It is entirely possible that some modulations of apparent size are mediated solely by higher-level brain regions, but are not represented in early visual cortex. These higher-level areas are of course likely to be involved even in our experiments. Nonetheless, differences in stimulus representations caused by idiosyncratic spatial tuning should be inherited by areas downstream the visual hierarchy, such as V2 and V3. In fact, we observed similar correlations between perceptual biases and pRF spread in V2 and V3. This is unsurprising given the pRF spreads across these early visual areas are also strongly correlated (though interestingly the surface areas of these regions are far less linked ${ }^{35}$ ). Therefore, signals in these regions may also be used for perceptual judgments. However, V1 would be a natural candidate for size estimates given it is the region with the smallest receptive fields and thus the finest spatial resolution. Future research must explore the neural substrate of size judgments, in particular with regards to where in the brain the sensory input is integrated into a perceptual decision ${ }^{1}$. Interestingly, topographically organized tuning for visual object size has recently been reported in parietal cortex ${ }^{40}$.

Our present findings imply that measurements of functional architecture in early sensory cortex can predict individual differences not only of objective discrimination abilities but also our subjective experience of the world. Theoretically, the principle discovered here should also apply to other sensory modalities, such as tuning for auditory frequency or tactile position, and may generalize to more complex forms of tuning, such as object identity or numerosity ${ }^{41}$.

\section{Methods}

Observers. The authors and several naive observers participated in these experiments. All observers were healthy and had normal or corrected-to-normal visual acuity. All observers gave written informed consent and procedures were approved by the UCL Research Ethics Committee.

Ten observers ( 4 authors; 3 female; 2 left-handed; ages $24-37$ years) took part in both the first behavioural experiment measuring perceptual biases at three eccentricities and in the fMRI retinotopic mapping experiment (henceforth, sizeeccentricity bias experiment). An additional 3 observers ( 1 female; all right-handed; ages 20-25 years) took part in behavioural experiments only but could not be recruited for the fMRI sessions (which commenced several months later and were 
conducted over the course of a year). These fMRI data also form part of a different study investigating the inter-session reliability of $\mathrm{pRF}$ analysis that we are preparing for a separate publication. Nine of the observers from the sizeeccentricity bias experiment ( 3 authors; 3 female; 1 left-handed; ages $25-37$ years at second test) took part in an additional behavioural experiment $\sim 1$ year after the first measuring perceptual biases in size perception (long-term bias reliability). Four observers ( 4 authors, 1 female, all right-handed; ages 33-38 years) participated in another experiment 2 years after the main experiment to again assess the reliability of bias estimates and also test a greater range of eccentricities (size far eccentricity bias). Six observers ( 5 authors; 2 female; all right-handed; ages 21-36 years) participated in the dichoptic control experiment (dichoptic bias).

General psychophysical procedure. Observers were seated in a dark, noiseshielded room in front of a computer screen (Samsung 2233RZ) using its native resolution of $1680 \times 1050$ pixels and a refresh rate of $120 \mathrm{~Hz}$. Minimum and maximum luminance values were 0.25 and $230 \mathrm{~cd} \mathrm{~m}^{-2}$. Head position was held at $48 \mathrm{~cm}$ from the screen with a chinrest. Observers used both hands to indicate responses by pressing buttons on a keyboard.

The dichoptic control experiment took place in a different testing room, using an Asus VG278 27" LCD monitor running its native resolution of $1920 \times 1080$ pixels and a refresh rate of $120 \mathrm{~Hz}$. Minimum and maximum luminance values were 0.16 and $100 \mathrm{~cd} \mathrm{~m}^{-2}$, with a viewing distance of $60 \mathrm{~cm}$ ensured with a chinrest. To produce dichoptic stimulation observers wore nVidia 3D Vision 2 shutter goggles synchronized with the refresh rate of the monitor. Frames for left and right eye stimulation thus alternated at $120 \mathrm{~Hz}$.

Multiple alternatives perceptual search (MAPS) procedure. To estimate perceptual biases efficiently at four visual field locations we developed the MAPS procedure. This is a matching paradigm using analyses related to reverse correlation or classification image approaches ${ }^{22,23}$ that seeks to directly estimate the points of subjective equality, whilst also allowing an inference of discrimination ability.

Stimuli. All stimuli were generated and displayed using MATLAB (The MathWorks Inc., Natick, MA) and the Psychophysics Toolbox version 3 (ref. 42). The stimuli in all the size discrimination experiments comprised light grey $\left(54 \mathrm{~cd} \mathrm{~m}^{-2}\right)$ circle outlines presented on a black background. Each stimulus array consisted of five circles (Fig. 1b). One, the reference, was presented in the centre of the screen and was always constant in size (diameter: $0.98^{\circ}$ visual angle). The remaining four, the targets, varied in size from trial to trial and independently from each other. They were presented at the four diagonal polar angles and at a distance of $3.92^{\circ}$ visual angle from the reference, except for the size-eccentricity bias experiment where target eccentricity could be $1.96^{\circ}, 3.92^{\circ}$ or $7.84^{\circ}$ visual angle and the size far eccentricity bias experiment where there were two additional eccentricities in the periphery $\left(11.76^{\circ}\right.$ and $\left.15.68^{\circ}\right)$. To measure the bias under the Delboeuf illusion, a larger inducer circle (diameter: $2.35^{\circ}$ ) surrounded each of the four target circles (but not the reference) to produce a contextual modulation of apparent size.

In all experiments, the independent variable (the stimulus dimension used to manipulate each of the targets) was the binary logarithm of the ratio of diameters for the target relative to the reference circles. In the size-eccentricity bias experiment only, the sizes of the four targets were drawn without replacement from a set of fixed sizes $(0, \pm 0.05, \pm 0.1, \pm 0.15, \pm 0.2, \pm 0.25, \pm 0.5, \pm 0.75$ or $\pm 1 \log$ units). Thus, frequently there was no 'correct' target to choose from. Because this made the task feel quite difficult for many observers, in subsequent experiments (long-term reliability and dichoptic bias) we decided to select a random subset of three targets from a Gaussian noise distribution centred on 0 (the size/orientation of the reference), while one target was correct, that is, it was set to 0 . The s.d. of the Gaussian noise was $0.3 \log$ units for size discrimination experiments.

Tasks. Each trial started with $500 \mathrm{~ms}$ during which only a fixation dot (diameter: $0.2^{\circ}$ ) was visible in the middle of the screen. This was followed by presentation of the stimulus array for $200 \mathrm{~ms}$ after which the screen returned to the fixation-only screen. Observers were instructed to make their response by pressing the F, V, K or $\mathrm{M}$ button on the keyboard corresponding to which of the four targets appeared most similar to the reference. After their response a 'ripple' effect over the target they had chosen provided feedback about their response. In the size discrimination experiments this constituted three $50 \mathrm{~ms}$ frames in which a circle increased in diameter from $0.49^{\circ}$ in steps of $0.33^{\circ}$ and in luminance.

Moreover, the colour of the fixation dot also changed during these $150 \mathrm{~ms}$ to provide feedback about whether the behavioural response was correct. In the sizeeccentricity bias experiment, the colour was green and slightly larger $\left(0.33^{\circ}\right)$ for correct trials and red for incorrect trials. In all later experiments, we only provided feedback on correct trials. This helped to reduce the anxiety associated with large numbers of incorrect trials that are common in this task: Accuracy was typically around $45-50 \%$ correct. Even though this is well in excess of chance performance of $25 \%$ it means that observers would frequently make mistakes.

Experimental runs were broken up into blocks of 20 trials. After each block there was a resting break. A message on the screen reminded observers of the task and indicated how many blocks they had already completed. Observers initiated blocks with a button press.

Size-eccentricity bias experiment. Observers were recruited for two sessions on separate days. In each session they performed six experimental runs, three with only circles and three with the Delboeuf stimuli. Each run tested one of the three target eccentricities. Trials with different eccentricities were run in separate blocks to avoid confounding these measurements with differences in attentional deployment across different eccentricities. There were 10 blocks per experimental run. In the size far eccentricity bias experiment we only tested observers in one session on the five target eccentricities.

Long-term bias reliability experiment. Half of the experimental runs observers performed measured their baseline biases. The other half of the runs contained artificially induced biases: two of the four targets were altered subtly: one by adding and one by subtracting $0.1 \log$ units. Which two targets were altered was counterbalanced across observers, as was the order of experimental runs. Observers were recruited for only one session comprising four runs (two with artificial bias) plus an additional run measuring biases for the Delboeuf stimuli. There were 10 blocks per experimental run. Only the results of the baseline biases (that is, without artificially induced bias) are presented in the present study. The remainder of these experiments form part of another study and will be presented elsewhere.

Dichoptic bias experiment. There were three experimental conditions in this experiment. By means of shutter goggles the stimulus arrays could be presented dichoptically, either binocularly or monocularly to either eye. To aid stereoscopic fusion we additionally added 5 concentric squares surrounding the stimulus arrays (side length: $8.1-10.5^{\circ}$ in equal steps). The three experimental conditions were randomly interleaved within each experimental run. There were 34 blocks per run; however, in this experiment each block comprised only 12 trials. Observers performed two such runs within a single session.

Analysis. To estimate perceptual biases we fit a model to predict a given observer's behavioural response in each trial (Fig. 1c). For each target stimulus location a Gaussian tuning curve denoted the output of a 'neural detector'. The detector producing the strongest output determined the predicted choice. The model fitted the peak location $(\mu)$ and dispersion $(\sigma)$ parameters of the Gaussian tuning curves that minimized the prediction error across all trials. Model fitting employed the Nelder-Mead simplex search optimization procedure ${ }^{43}$. We initialized the $\mu$ parameter as the mean stimulus value (offset in logarithmic size ratio from 0 ) whenever a given target location was chosen incorrectly. We initialized the $\sigma$ parameter as the s.d. across all stimulus values when a given target location was chosen. The final model-fitting procedure, however, always used all trials, correct and incorrect.

Retinotopic mapping experiment. The same 10 observers from the size-eccentricity bias experiment participated in two sessions of retinotopic mapping in a Siemens Avanto 1.5 T MRI scanner using a 32-channel head coil located at the Birkbeck-UCL Centre for Neuroimaging. The front half of the coil was removed to allow unrestricted field of view leaving 20 channels. Observers lay supine and watched the mapping stimuli, which were projected onto a screen (resolution: $1,920 \times 1,080)$ at the back of the bore, via a mirror mounted on the head coil. The viewing distance was $68 \mathrm{~cm}$.

We used a $\mathrm{T} 2^{*}$-weighted multiband two-dimensional (2D) echo-planar sequence $^{44}$ to acquire 235 functional volumes per pRF mapping run and 310 volumes for a run to estimate the hemodynamic response function (HRF). In addition, we collected a T1-weighted anatomical magnetization-prepared rapid acquisition with gradient echo (MPRAGE) scan with $1 \mathrm{~mm}$ isotropic voxels $(\mathrm{TR}=2,730 \mathrm{~ms}, \mathrm{TE}=3.57 \mathrm{~ms})$ using the full 32 -channel head coil.

The method we used for analysing $\mathrm{pRF}^{32-34}$. We used a combined wedge and ring stimulus that contained natural images that changed twice a second. The MATLAB toolbox (available at http://dx.doi.org/10.6084/m9.figshare.1344765) models the $\mathrm{pRF}$ of each voxel as a 2D Gaussian in visual space and incorporates the hemodynamic response function measured for each individual observer. It determines the visual field location ( $x$ and $y$ in Cartesian coordinates) and the spread (s.d.) of the pRF plus an overall response amplitude.

Stimuli and task. A polar wedge subtending a polar angle of $12^{\circ}$ rotated in 60 discrete steps (one per second) around the fixation dot (diameter: $0.13^{\circ}$ surrounded by a $0.25^{\circ}$ annulus where contrast ramped up linearly). A ring expanded or contracted, both in width and overall diameter, in 36 logarithmic steps. The maximal eccentricity of the wedge and ring was $8.5^{\circ}$. There were three cycles of wedge rotation and five cycles of ring expansion/contraction. Each mapping run concluded with $45 \mathrm{~s}$ of a fixation-only period. At all times a low contrast 'radar screen' pattern (Fig. 2a) was superimposed on the screen to aid fixation compliance.

The wedge and ring parts contained colourful natural images (Fig. 2a) from Google Image search, which changed every $500 \mathrm{~ms}$. They depicted outdoor scenes (tropical beaches, forests, mountains and rural landscapes), faces, various animals and pictures of written script (228 images in total). One picture depicted the 'Modern Anderson' clan tartan. These pictures were always rotated in accordance with the current orientation of the wedge. Observers were asked to fixate a fixation dot at all times. With a probability of 0.03 every $200 \mathrm{~ms}$ the black fixation dot 
would change colour for $200 \mathrm{~ms}$ to one of the primary and complementary colours or white followed by another $200 \mathrm{~ms}$ of black. Observers were asked to tap their finger when the dot turned red. To also maintain attention on the mapping stimulus they were asked to tap their finger whenever they saw the tartan image.

In alternating runs the wedge rotated in clockwise and counterclockwise directions, while the ring expanded and contracted, respectively. In each session we collected six such mapping runs and an additional run to estimate the hemodynamic response function. The latter contained 10 trials each of which started with a 2-s sequence of four natural images from the same set used for mapping. These were presented in a circular aperture centred on fixation with radius $8.5^{\circ}$ visual angle. This was followed by $28 \mathrm{~s}$ of the blank screen (fixation and radar screen only).

Preprocessing and pRF modelling. Functional MRI data were first preprocessed using SPM8 (Wellcome Trust Centre for Neuroimaging, London, http://www.fil.ion.ucl.ac.uk/spm/software/spm8). The first 10 volumes were removed to allow the signal to reach equilibrium. We performed intensity bias correction, realignment and unwarping, and coregistration of the functional data to the structural scan, all using default parameters. We used FreeSurfer (https://surfer.nmr.mgh.harvard.edu/fswiki) for automatic segmentation and reconstruction to create a three-dimensional inflated model of the cortical surfaces for the grey-white matter boundary and the pial surface, respectively $y^{45,46}$. We then projected the functional data to the cortical surface by finding for each vertex in the surface mesh the median position between the grey-white matter and pial surfaces in the functional volume. All further analyses were performed in surface space.

We applied linear detrending to the time series from each vertex in each run and then $z$-standardized them. Alternating pRF mapping runs (that is, those sharing the same stimulus directions-clockwise/expanding and counterclockwise/ contracting) were averaged. These two average runs were then concatenated. We further divided the HRF run into the 10 epochs and averaged them. Only vertexes for which the average response minus the s.e. in the first half of the trial was larger than zero were included. The HRFs for these vertices were than averaged and we fit a two-gamma function with four free parameters: the amplitude, the peak latency, the undershoot latency, and the ratio amplitude between peak and undershoot.

Population receptive field analysis was conducted in a two-stage procedure. First, a coarse fit was performed on data smoothed with a large kernel on the spherical surface (FWHM (full-width at half maximum) $=5 \mathrm{~mm}$ ). We performed an extensive grid search on every permutation of 15 plausible values for $x$ and $y$, respectively, and a range of pRF spreads from $0.18^{\circ}$ to $17^{\circ}$ in 34 logarithmic steps (0.2 in binary logarithm). For each permutation we generated a predicted time series by calculating the overlap between a $2 \mathrm{D}$ Gaussian pRF profile and a binary aperture of the mapping stimulus for every volume. This time series was then $z$ standardized and convolved with the subject-specific HRF. The grid search is a very fast operation that computes the set of three pRF parameters that produce the maximal Pearson's correlation between the predicted and observed time series for the whole set of search grid parameters and all vertices. This was followed by the slow fine fit. Here we used the parameters identified by the coarse fit to seed an optimization algorithm ${ }^{43,47}$ on a vertex by vertex basis to refine the parameter estimates by minimizing the squared residuals between the predicted and observed time series. This stage used the unsmoothed functional data and also included a fourth amplitude parameter to estimate response strength. Finally, the estimates parameter maps were also smoothed on the spherical surface with a modest kernel $(\mathrm{FWHM}=3 \mathrm{~mm})$

Analysis of functional cortical architecture. We next delineated the early visual regions (specifically V1) manually based on reversals in the polar angle map and the extent of the activated portion of visual cortex along the anterior-posterior axis. We then extracted the pRF parameter data separately from each visual field quadrant represented in V1. Data were divided into eccentricity bands $1^{\circ}$ in width starting from $1^{\circ}$ eccentricity up to $9^{\circ}$. For each eccentricity band we then calculated mean pRF spread and the sum of surface area estimates. For pRF spread we used the raw, unsmoothed $\mathrm{pRF}$ spread estimates produced by our fine-fitting procedure. However, the quantification of surface area requires a smooth gradient in the eccentricity map without any gaps in the map and with minimal position scatter in pRF positions. Therefore, we used the final smoothed parameter maps for this analysis. The results for pRF spread are very consistent when using smoothed parameter maps, but we reasoned that the unsmoothed data make fewer assumptions.

To extract the parameters for each stimulus location we fit polynomial functions to the relationship between these binned parameters and eccentricity. For pRF spread we used a first-order polynomial (that is, a linear relationship). For surface area we used a second order polynomial. We then interpolated each person's pRF spread/surface area at the 12 target locations in the behavioural experiments (that is, 4 stimulus locations and 3 eccentricities. Individual plots for each observer and visual field quadrant are included in the Supplementary Information. We also quantified the macroscopic surface area of each visual field quadrant in $\mathrm{V} 1$ by summing the surface area between $1^{\circ}$ and $9^{\circ}$. This range ensured that artifactual, noisy estimates in the foveal confluence or edge effects well beyond the stimulated region did not introduce spurious differences between individuals. In our main analyses we normalized all surface area measures relative to the whole cortical surface area. However, results are also very consistent for using the square root of the absolute surface area for this analysis.

Data availability. Materials and behavioural data: http://dx.doi.org/10.6084/ m9.figshare.1579442.

Processed pRF data per observer: http://dx.doi.org/10.5281/zenodo.19150. All other data supporting these findings is available from the corresponding author on request.

\section{References}

1. Schwarzkopf, D. S. Where is size in the brain of the beholder? Multisens. Res. 28, 285-296 (2015).

2. Murray, S. O., Boyaci, H. \& Kersten, D. The representation of perceived angular size in human primary visual cortex. Nat. Neurosci. 9, 429-434 (2006).

3. Fang, F., Boyaci, H., Kersten, D. \& Murray, S. O. Attention-dependent representation of a size illusion in human V1. Curr. Biol. 18, 1707-1712 (2008).

4. Sperandio, I., Chouinard, P. A. \& Goodale, M. A. Retinotopic activity in V1 reflects the perceived and not the retinal size of an afterimage. Nat. Neurosci. 15, 540-542 (2012).

5. Pooresmaeili, A., Arrighi, R., Biagi, L. \& Morrone, M. C. Blood oxygen level-dependent activation of the primary visual cortex predicts size adaptation illusion. J. Neurosci. 33, 15999-16008 (2013).

6. Ni, A. M., Murray, S. O. \& Horwitz, G. D. Object-centered shifts of receptive field positions in monkey primary visual cortex. Curr. Biol. 24, 1653-1658 (2014).

7. He, D., Mo, C., Wang, Y. \& Fang, F. Position shifts of fMRI-based population receptive fields in human visual cortex induced by Ponzo illusion. Exp. Brain Res. 233, 3535-3541 (2015).

8. Schwarzkopf, D. S., Song, C. \& Rees, G. The surface area of human V1 predicts the subjective experience of object size. Nat. Neurosci. 14, 28-30 (2011).

9. Schwarzkopf, D. S. \& Rees, G. Subjective size perception depends on central visual cortical magnification in human v1. PLOS ONE 8, e60550 (2013).

10. Song, C. et al. Effective connectivity within human primary visual cortex predicts interindividual diversity in illusory perception. J. Neurosci. 33, 18781-18791 (2013).

11. Song, C., Schwarzkopf, D. S. \& Rees, G. Variability in visual cortex size reflects tradeoff between local orientation sensitivity and global orientation modulation. Nat. Commun. 4, 2201 (2013).

12. Genç, E., Bergmann, J., Singer, W. \& Kohler, A. Surface area of early visual cortex predicts individual speed of traveling waves during binocular rivalry. Cereb. Cortex 25, 1499-1508 (2014).

13. Verghese, A., Kolbe, S. C., Anderson, A. J., Egan, G. F. \& Vidyasagar, T. R. Functional size of human visual area V1: A neural correlate of top-down attention. NeuroImage 93 Pt 1, 47-52 (2014).

14. Bergmann, J., Genç, E., Kohler, A., Singer, W. \& Pearson, J. Neural anatomy of primary visual cortex limits visual working memory. Cereb. Cortex 26, 43-50 (2014).

15. Bergmann, J., Genç, E., Kohler, A., Singer, W. \& Pearson, J. Smaller primary visual cortex is associated with stronger, but less precise mental imagery. Cereb. Cortex. doi:10.1093/cercor/bhv186 (18 August 2015).

16. Duncan, R. O. \& Boynton, G. M. Cortical magnification within human primary visual cortex correlates with acuity thresholds. Neuron 38, 659-671 (2003).

17. Song, C., Schwarzkopf, D. S., Kanai, R. \& Rees, G. Neural population tuning links visual cortical anatomy to human visual perception. Neuron 85, 641-656 (2015)

18. Helmholtz, H. in Handbuch der Physiologischen Optik (Leipzig : Leopold Voss, 1867).

19. Newsome, L. R. Visual angle and apparent size of objects in peripheral vision. Percept. Psychophys. 12, 300-304 (1972).

20. Anstis, S. Picturing peripheral acuity. Perception 27, 817-825 (1998).

21. Afraz, A., Pashkam, M. V. \& Cavanagh, P. Spatial heterogeneity in the perception of face and form attributes. Curr. Biol. 20, 2112-2116 (2010).

22. Abbey, C. K. \& Eckstein, M. P. Classification image analysis: estimation and statistical inference for two-alternative forced-choice experiments. J. Vis. 2, 66-78 (2002)

23. Li, R. W., Levi, D. M. \& Klein, S. A. Perceptual learning improves efficiency by re-tuning the decision 'template' for position discrimination. Nat. Neurosci. 7, $178-183$ (2004).

24. Morgan, M., Dillenburger, B., Raphael, S. \& Solomon, J. A. Observers can voluntarily shift their psychometric functions without losing sensitivity. Atten. Percept. Psychophys. 74, 185-193 (2012).

25. Morgan, M. J., Melmoth, D. \& Solomon, J. A. Linking hypotheses underlying Class A and Class B methods. Vis. Neurosci. 30, 197-206 (2013).

26. Jogan, M. \& Stocker, A. A. A new two-alternative forced choice method for the unbiased characterization of perceptual bias and discriminability. J. Vis. 14, 20 (2014).

27. Bedell, H. E. \& Johnson, C. A. The perceived size of targets in the peripheral and central visual fields. Ophthalmic. Physiol. Opt. 4, 123-131 (1984).

28. Delboeuf, J. Sur une nouvelle illusion d'optique. Acade Mie R. Sci. Lett. B arts Belg. Bull. 24, 545-558 (1892). 
29. van den Berg, R., Roerdink, J. B. T. M. \& Cornelissen, F. W. On the generality of crowding: visual crowding in size, saturation, and hue compared to orientation. J. Vis. 7, 14.1-11 (2007).

30. Abrams, J., Nizam, A. \& Carrasco, M. Isoeccentric locations are not equivalent: the extent of the vertical meridian asymmetry. Vis. Res. 52, 70-78 (2012).

31. Rubin, N., Nakayama, K. \& Shapley, R. Enhanced perception of illusory contours in the lower versus upper visual hemifields. Science 271, 651-653 (1996).

32. Dumoulin, S. O. \& Wandell, B. A. Population receptive field estimates in human visual cortex. Neuroimage 39, 647-660 (2008).

33. Schwarzkopf, D. S., Anderson, E. J., de Haas, B., White, S. J. \& Rees, G. Larger extrastriate population receptive fields in autism spectrum disorders. J. Neurosci. 34, 2713-2724 (2014).

34. Alvarez, I., De Haas, B. A., Clark, C. A., Rees, G. \& Schwarzkopf, D. S. Comparing different stimulus configurations for population receptive field mapping in human fMRI. Front. Hum. Neurosci. 9, 96 (2015).

35. Dougherty, R. F. et al. Visual field representations and locations of visual areas $\mathrm{V} 1 / 2 / 3$ in human visual cortex. J. Vis. 3, 586-598 (2003).

36. Harvey, B. M. \& Dumoulin, S. O. The relationship between cortical magnification factor and population receptive field size in human visual cortex: constancies in cortical architecture. J. Neurosci. 31, 13604-13612 (2011).

37. Pouget, A., Dayan, P. \& Zemel, R. Information processing with population codes. Nat. Rev. Neurosci. 1, 125-132 (2000).

38. Treue, S., Hol, K. \& Rauber, H.-J. Seeing multiple directions of motion-physiology and psychophysics. Nat. Neurosci. 3, 270-276 (2000).

39. Kay, K. N., Winawer, J., Mezer, A. \& Wandell, B. A. Compressive spatial summation in human visual cortex. J. Neurophysiol. 110, 481-494 (2013).

40. Harvey, B. M., Fracasso, A., Petridou, N. \& Dumoulin, S. O. Topographic representations of object size and relationships with numerosity reveal generalized quantity processing in human parietal cortex. Proc. Natl Acad. Sci. USA 112, 13525-13530 (2015).

41. Harvey, B. M., Klein, B. P., Petridou, N. \& Dumoulin, S. O. Topographic representation of numerosity in the human parietal cortex. Science 341, 1123-1126 (2013).

42. Brainard, D. H. The psychophysics toolbox. Spat. Vis. 10, 433-436 (1997).

43. Lagarias, J., Reeds, J., Wright, M. \& Wright, P. Convergence properties of the Nelder-Mead simplex method in low dimensions. SIAM J. Optim. 9, 112-147 (1998).

44. Breuer, F. A. et al. Controlled aliasing in parallel imaging results in higher acceleration (CAIPIRINHA) for multi-slice imaging. Magn. Reson. Med. 53, 684-691 (2005)

45. Dale, A. M., Fischl, B. \& Sereno, M. I. Cortical surface-based analysis. I. Segmentation and surface reconstruction. Neuroimage 9, 179-194 (1999).
46. Fischl, B., Sereno, M. I. \& Dale, A. M. Cortical surface-based analysis. II: Inflation, flattening, and a surface-based coordinate system. Neuroimage 9, 195-207 (1999).

47. Nelder, J. A. \& Mead, R. A Simplex Method for Function Minimization. Comput. J. 7, 308-313 (1965).

\section{Acknowledgements}

This research was supported by an ERC Starting Grant (310829) to D.S.S., a Research Fellowship by the Deutsche Forschungsgemeinschaft (Ha 7574/1-1) to B.d.H., a UCL Graduate School Bridging Fund and a Wellcome Trust Institutional Strategic Support Fund to C.M. and a UK MRC Career Development Award (MR/K024817/1) to J.A.G.

\section{Author contributions}

Conception and design: D.S.S., B.d.H., J.A.G., A.P., A.B. Data collection: C.M., B.d.H., J.A.v.D., A.P., A.B. Data analysis: D.S.S., J.A.v.D., B.d.H. Manuscript writing: D.S.S. wrote the initial draft, but all authors contributed to the final manuscript.

\section{Additional information}

Supplementary Information accompanies this paper at http://www.nature.com/ naturecommunications

Competing financial interests: The authors declare no competing financial interests.

Reprints and permission information is available online at http://npg.nature.com/ reprintsandpermissions/

How to cite this article: Moutsiana, C. et al. Cortical idiosyncrasies predict the per ception of object size. Nat. Commun. 7:12110 doi: 10.1038/ncomms12110 (2016).

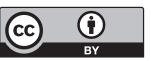

This work is licensed under a Creative Commons Attribution 4.0 International License. The images or other third party material in this article are included in the article's Creative Commons license, unless indicated otherwise in the credit line; if the material is not included under the Creative Commons license, users will need to obtain permission from the license holder to reproduce the material. To view a copy of this license, visit http://creativecommons.org/licenses/by/4.0/

(C) The Author(s) 2016 